\title{
Influence of crystal-melt interface shape on self-seeding and single crystalline quality
}

\author{
D B GADKARI*, P SHASHIDHARAN ${ }^{\dagger}, \mathrm{K} B$ LAL $^{\dagger}$ and B M ARORA \\ *Department of Physics, Mithibai College, Mumbai 400 056, India \\ 'Department of Physics, University of Mumbai, Mumbai 400 098, India \\ ${ }^{\ddagger}$ CMPS \& MS, Tata Institute of Fundamental Research, Mumbai 400 005, India
}

MS received 14 December 2000; revised 22 June 2001

\begin{abstract}
The growth of Sb-based crystals (InSb, GaSb etc) was undertaken using resistive heater furnace by vertical directional solidification (VDS) technique. Crystal-melt interface shape during the growth was shown to convert from concave to convex along the crystal axis of the ingots. Many antimonide (Sb) crystals of $8 \mathrm{~mm}$ to $18 \mathrm{~mm}$ diameter were grown by optimized growth parameters. The forced convection and absence of conducting support to ampoule showed improvement in crystal quality of as grown ingots. Crystals showed preferred orientation and self-seeding. Results on interface shape and crystallinity of ingots were found to be in good agreement with the experiments.
\end{abstract}

Keywords. Self-seeding; orientation; interface shape; antimonide crystals; VDS technique.

\section{Introduction}

The role of semi-conductor crystals in commercial applications becomes dominant with time. Device manufacturer is demanding a high quality and large single crystals for device fabrication and its industrial yield (Thomas et al 1993; Marie Mayer 1998). Therefore, several research groups are engaged in improving crystal growth systems (Lopez et al 1999). On this basis and the fundamental characteristics of source materials, various crystal growth models have been proposed for the uplift of crystal growth techniques. However, basic crystal growth techniques from melt are Czochralski (de Oliveira and de Carvalho 1995), Bridgman (Althaus et al 1996), floating zone (Herrmann and Muller 1995) and vertical gradient freeze (Aman et al 1996). Each technique has an inherent crystal growth problem. In order to obtain solutions for the problems, crystal growth experts have concentrated on mass transport (Neugebauer et al 1991), heat transfer (Suzuli et al 1993), crystal-melt interface (Boiten et al 1998) and growth modelling (Lan and Ting 1995). In literature, only a few references are cited on the growth experiments by solidification (Derebail et al 1992) in ampoule with a conical at one end without seed (selfseeding) (Ivanov 1998). Moreover, the dimensions and geometrical shape of the conical region of ampoule play very important role on the crystal growth (single crystal growth).

\footnotetext{
*Author for correspondence
}

Further, temperature gradient is not employed on the crucible or ampoule wall in the aforementioned systems. However, it is considered on the external support, which holds the ampoule in heating furnace. In VDS technique (Gadkari et al 1999a), this problem has been eliminated by not having support to the ampoule downward but a shaft into growth chamber hangs it. Ampoule can be rotated clock/anti-clock wise as well as lowered/pulled up in a controlled manner (Gadkari et al 1999b). According to the source materials physical and chemical properties, optimized growth conditions (Gadkari et al 1998a) and modification in growth process have adjusted in experimental setup. Moreover, a homogeneous and high quality single crystal of binary (Gadkari et al 1998b, 1999b) and ternary compound (Gadkari et al 1999c) are possible by VDS technique.

The aim of the present paper is to analyse the growth conditions and crystal-melt interface shape. Influence of the crystal-melt interface on the single crystal growth of the antimonide crystals has also been discussed.

\section{Experimental}

The growth of undoped (InSb, GaSb), doped (InSb : Te, GaSb : Al) and alloyed (InGaSb, InTlSb, GaInSb etc) (Gadkari et al 1999) Sb-based semiconductor crystal was performed by VDS-technique (Gadkari et al 1999a). Main features of the growth are briefed as follows: high quality $\mathrm{In}, \mathrm{Ga}, \mathrm{Sb}$ and $\mathrm{Te}, \mathrm{Tl}$ etc $(6 \mathrm{~N})$ were used as the source materials. For the undoped growth, source materials were 
taken in stoichiometric proportion and kept in quartz ampoule (conical at one end). Doped crystals were grown by mixing dopants into undoped charge for the respective growths before sealing. Similarly, various alloyed growths were performed for the ternary crystals. The quartz ampoule with a charge was evacuated ( $10{ }^{5}$ torr), back filling with argon alternately (10 times), and then sealed by refilling argon (350 torr). The charge in the ampoule was melted at $850^{\circ} \mathrm{C}$ and kept at this temperature for $12 \mathrm{~h}$ for synthesis and homogeneous mixing of melt composition. Afterwards, ampoule was lowered $(10 \mathrm{~mm} / \mathrm{h}, 10 \mathrm{rpm})$ to the temperature $50^{\circ} \mathrm{C}$ above the melting temperature of the crystals (InSb, m.p. $530^{\circ} \mathrm{C}$ and $\mathrm{GaSb}, 712^{\circ} \mathrm{C} \mathrm{m.p.)} \mathrm{and}$ maintained at this position for $12 \mathrm{~h}$, for thermal stabilization of the melt before proceeding with the crystal growth. Growth of semiconductor crystals was initiated by lowering ampoule from hot zone at translation speed of $3 \mathrm{~mm} / \mathrm{h}$ and rotation rate $10 \mathrm{rpm}$ till the last drop of melt i.e. last to freeze part (LFP), came out from the hot zone. Later on, the ampoule was lowered $(10 \mathrm{~mm} / \mathrm{h}, 10 \mathrm{rpm})$ to $300^{\circ} \mathrm{C}$ and then ingot temperature was held constant at $300^{\circ} \mathrm{C}$ for $18-20 \mathrm{~h}$ prior to removal from the growth chamber.

From the as grown crystal, disks were cut perpendicular to the grown axis (thickness of the order of $500 \mu \mathrm{m}$ ). Substrates were cleaned, lapped and polished (Gadkari et al 1998a) to mirror finish. Characterization techniques such as XRD, electrical, optical and chemical etching were performed on the substrates cut from the different sections of the ingots. Furthermore, these measurements were analysed for the influence of crystal-melt interface on selfseeding and crystallinity of the as grown crystals.

\section{Results and discussion}

\subsection{As grown crystals and interface shape}

VDS technique was used for the growth of undoped, doped and alloyed crystals. Typical ingot of InSb 17 (growth no. 17), GaSb-4 (growth no. 4) and InGaSb3 (growth no. 3) are shown in figures 1a, b, c, respectively. In preliminary observation, the faceted growth is visible in the neck and shoulder region indicating the surface to be free and not wetting the quartz crucible i.e. melt did not adhere to the walls of the ampoule. The first to freeze part (FFP) is slightly concave since the reduction in crosssectional area of conical quartz ampoule acts as a thermal choke to the downward axial heat flow. However, delamination is replicated in the same ingot surface with no polycrystalline nucleation. Our effort to match the thermal conductivity of the ampoule with air surrounding to that of the solidified crystal may have flattened the interface so that preferred orientation is promoted and it becomes self-seeding region. Therefore, orientation and selfseeding growth (Gadkari et al 1998a) have been propa- gated in the ingots grown using VDS technique but it was not a smooth transition. LFP is fully composed with no excess of source element, which indicated that the compositional control on the crystal growth from the melt was effective. This is due to the effect on the crystal-melt interface shape, which initially was concave then it shifted to planar and finally converted into convex. This can be
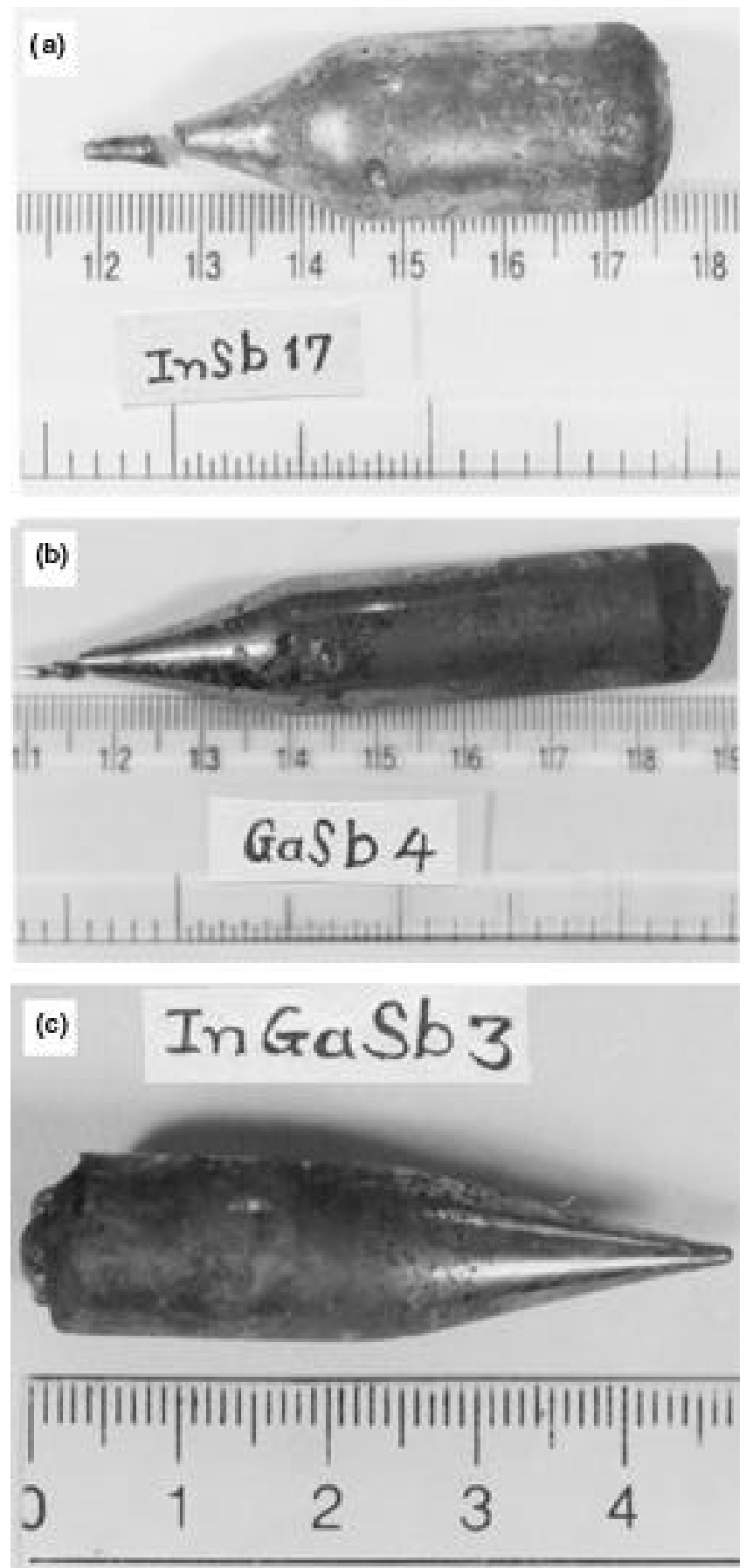

Figure 1. Typical as grown ingots: a. InSb17, b. GaSb-4 and c. InGaSb-3 represent the smooth and glossy surface without surface defects, indicating orientation growth due to selfseeding. 
examined from the visibility of the as grown crystals, and by the shape of FFP and LFP and the lack of interaction or de-wetting of the ampoule. This is also confirmed by the diameter of the ingots, which are smaller than that of the inner diameter of the quartz ampoule due to the volume shrinkage. This corresponds to the low axial and radial temperature gradients associated with the VDStechnique. Therefore, low level of thermo elastic stress may be developed with a low level defect density.

The shape of the crystal-melt interface at the intersection of three-phase boundary (TPB), and crystal-melt-ampoule wall, must remain steady. Ampoule wall influences edge faceting twin initiation and the nucleation of secondary growth orientation. In the literature it is recommended that planar or slightly convex interface shape is desirable for high degree of crystallinity. Normally, the thermal conductivity of the ampoule could affect the interface shape at TPB. If the thermal conductivity along the ampoule wall is greater than that of the crystal then, it tends to make the interface shape concave. Initially, crystal has less thermal conductivity due to the large aspect ratio (melt height/diameter). Therefore, the interface shape has concave shape with higher concavity as shown in figure 2 but as the crystal grow in length, the thermal conductivity increases and aspect ratio decreases with a result decrease in concavity. This effect introduces to change interface shape from concave-planar-convex as depicted in figure 2. This observation is seen in all the growth of crystals ranging in diameter $8 \mathrm{~mm}$ to $18 \mathrm{~mm}$. However, the GaSb crystals of $15 \mathrm{~mm}$ diameter (growth

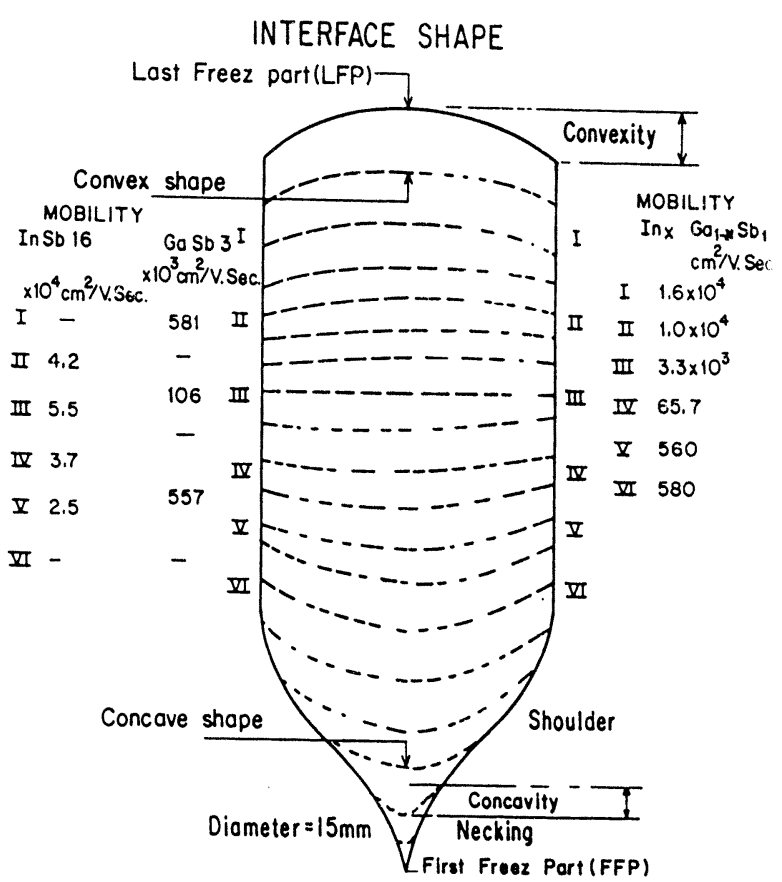

Figure 2. Schematic representation of crystal-melt interface in VDS technique. Interface shows the conversion of concave to convex shape along the crystal axis (growth axis). no. 4) and InSb crystals of $18 \mathrm{~mm}$ diameter (growth no. 17) are under characterization and results will be reported elsewhere.

\subsection{Single crystal growth}

On contracting growth condition, TPB and interface have been lead to the single crystals of binary and multinery by VDS technique. With these parameters, we have prevented either spurious nucleation of grain or sticking of the crystal to the wall of the ampoule. Single crystal growth necessitates the avoidance of any contact between ampoule wall and the growing crystals. This achievement has been confirmed by the smaller diameter of the as grown crystals. VDS technique has proved as the easiest way to reach this goal by adjusting/controlling the temperature gradient and maintaining crystal-melt interface steady. Glossy appearance of the ingots indicate single crystallinity. For confirmation, substrate had been characterized by XRD. Figures 3a, b represent the typical XRD pattern obtained for different growths. To study the single crystal growth, substrate from the FFP, middle and LFP part had been analysed. Typical XRD patterns for undoped InSb16 is shown in figure 3a, which has dominant (022) reflection. GaSb3 growth has also seen with prominent $(022)$ reflection (figure $3 a)$. Figure $3 b$ represents the XRD patterns for InSb (growth nos 8, 12 and 13), which also showed orientation growth. The growth of binary (InSb, GaSb) crystal showed XRD with FWHM of the order of 50-90 arc sec. But alloyed growth such as InTlSb, GaInSb, InGaSb and doped growth InSb : Te and GaSb : Al showed FWHM higher than the aforementioned data. Interestingly binary crystals have shown most of the growth with dominant (022) reflection. However, (022) reflection has been repeated by the substrate laterally cut to the growth axis in several growth. This data is attributed to the uniform orientation along the growth axis. Therefore, it is possible to grow preferred orientation using VDS technique by self-seeding, i.e. growth without seed. Moreover, orientation growth is also confirmed using Philips X'pert diffractometer, JEOL JSM-840 and SIEMENS Krystallo diffractometer. The XRD measurements showed higher counts at the middle part and lowest counts at the conical section of the ingots. This data can be related to the crystallinity order in the structure with that of the interface shape as shown in figure 2. Planar interface may be the cause of the higher counts at the middle, since interface shape variation speculated as concave-planar and convex with the FFP to LFP. Lower counts in conical section may be concave curvature of interface which is introducing slight structural disorder and slightly convex at LFP may be creating ordered structure with highest counts at the middle part. These measurements reveal the influence of the self-seed growth. Further, this data is also confirmed by the Hall-Van der 
Pauw electrical measurements which is represented by highest mobility of the middle section of the ingots as speculated for the planar interface shape (see figure 2) (Gadkari et al 1997, 1998a).

\subsection{Growth morphology}

Polished substrate of antimonide crystals of III-V semiconductors have etched in selective chemical etchant $\mathrm{CP} 4$ and modified CP4. Figures $4 \mathrm{a}, \mathrm{b}$ represent the microphotograph of undoped InSb 8-5 substrate. Similar micro features are seen in low crystallinity ingots with higher degree of crystallinity (ingots are nearly free from dislocation i.e. low dislocation density). Figure 4a shows a straight single oriented twin boundary (lamellae). Twinning is the constant problem in the III-V bond semiconductor single crystals. Normally, high degree of ionicity of III-V bond leads to a low stacking fault energy (structural disorder) that occurs in the homogeneously grown layer as a twin boundary. The twin boundary (lamellae) is straight and transverse to the growth axis. This reveals that transverse growth is a homogeneous layer with single orientation. Further, another probable cause of the twinning may be the inclusions or cluster of point defects and temperature fluctuations. These have direct effect on the interface. Therefore, the shape of the crystal-melt interface at TPB influence edge faceting, twin initiation and secondary orientation growth due to the nucleation. In our study, we have seen recession by (a)
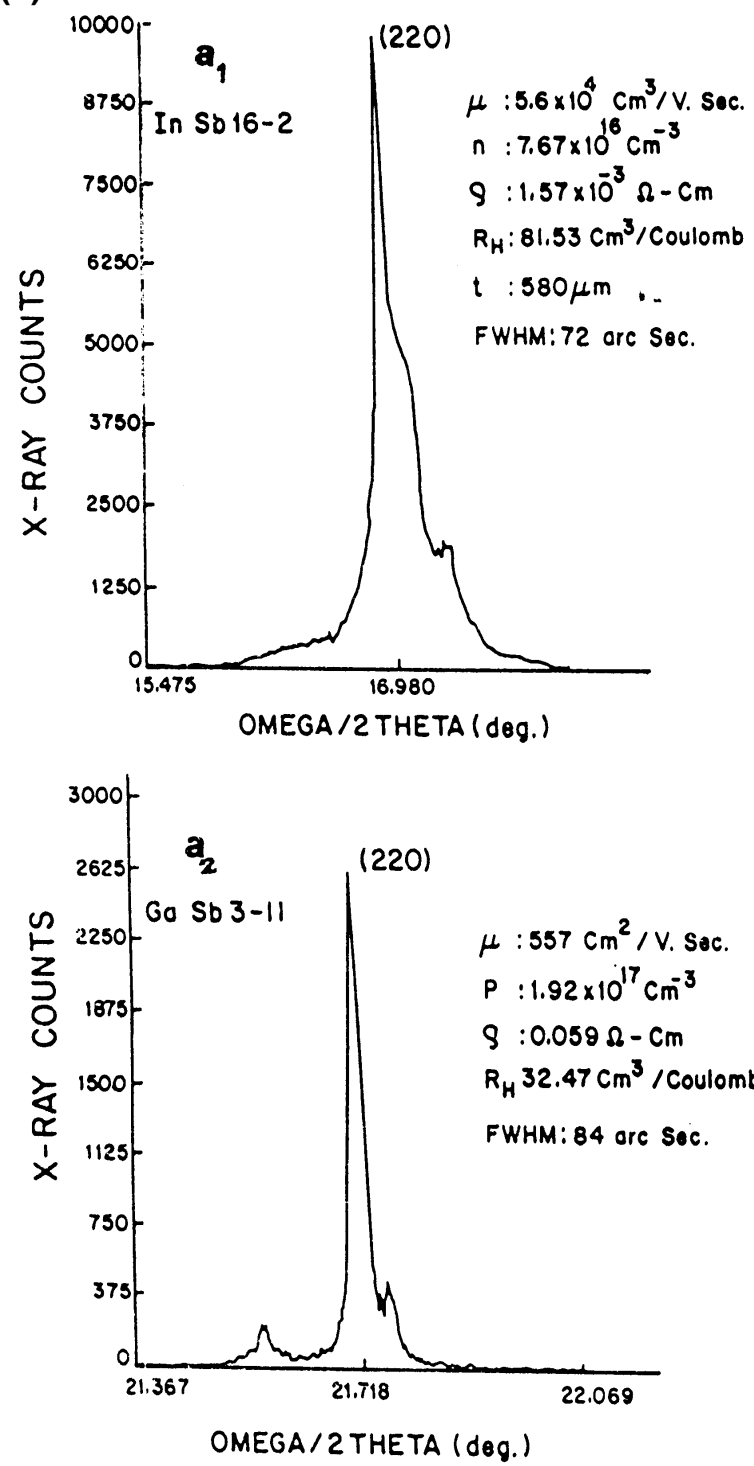

(b)
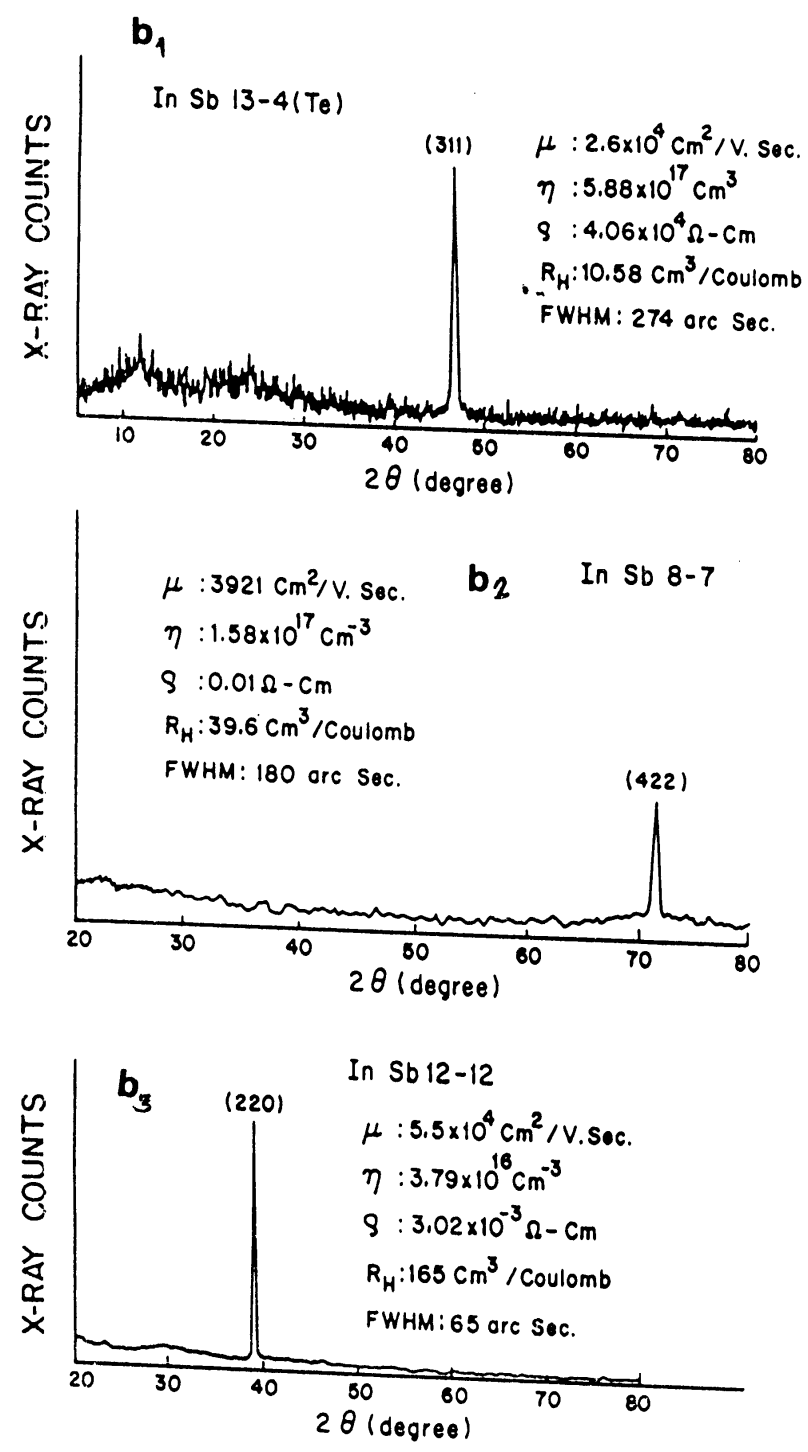

Figure 3 a. XRD pattern observed as the preferred orientation growth for undoped InSb16 and GaSb3. Generally, majority growth showed (022) reflection as the dominant orientation in VDS growth and $\mathbf{b}$. XRD pattern observed as the preferred orientation growth for undoped InSb12-12, InSb8-7 and Te-doped InSb13-4 as grown ingots. 
controlled growth conditions in VDS technique. GaSb has higher bond ionicity, therefore, lower is the stacking fault energy barrier and hence less twin and grain boundaries are observed, while InSb showed opposite properties to that of GaSb. Temperature fluctuation in melt can be another parameter influencing microstructures. This will lead to a series of irregularly spaced edge/surface effects i.e. multi facet growth. However, these facets result in twinning as shown in figure $4 \mathrm{a}$. Besides this, occasionally local stress may be generated at the TPB at the interface by the crystal sticking to the ampoule wall and later may result into second nucleation generation as the grain boundary seen in figure $4 \mathrm{~b}$ with multi orientation growth. Therefore, it is difficult to conclude here that either this microstructure (figure 4b) is a grain boundary or multi orientated twinning feature. Besides this, there are stress induced micro features observed in the conical portion of the ingots and may be different phases of the source materials intending congruent growth at initial stage. But these microstructures are reduced significantly in VDS tech- nique. This can be confirmed by (i) not wetting of the melt to ampoule wall, (ii) ingots came out easily, (iii) glossy surface of the ingots, and (iv) ingot diameter is smaller than the diameter of ampoule.

Figures 5a, b show the eutectic growth in alloyed crystals. Figure 5a shows the star like structure in InSb8-5 ingot. In this growth excess $\mathrm{Sb}$ was added as the isovalent material. The $\mathrm{Sb}$ rich region has been grown in homogeneous chain of $\mathrm{InSb}$ at higher temperature ( $\mathrm{Sb}$ m.p. $630^{\circ} \mathrm{C}$ ). There is a termination and extended growth of Sb-ridges but interpretation of star like behaviour of eutectic growth is difficult. However, Sb has higher melting point $\left(630^{\circ} \mathrm{C}\right)$ in comparison to $\mathrm{InSb}$ (m.p. $530^{\circ} \mathrm{C}$ ), which could be influenced by the constitutional supercooling as a result eutectic growth may be favoured. Interestingly, the eutectic micro features are absent in stoichiometric growth of InSb. Figure $5 \mathrm{~b}$ shows that microstructures are developed in heavily Te-doped InSb9-6 growth. The striations are favoured in this growth by the fluctuation in impurity and incorporation of it. Some
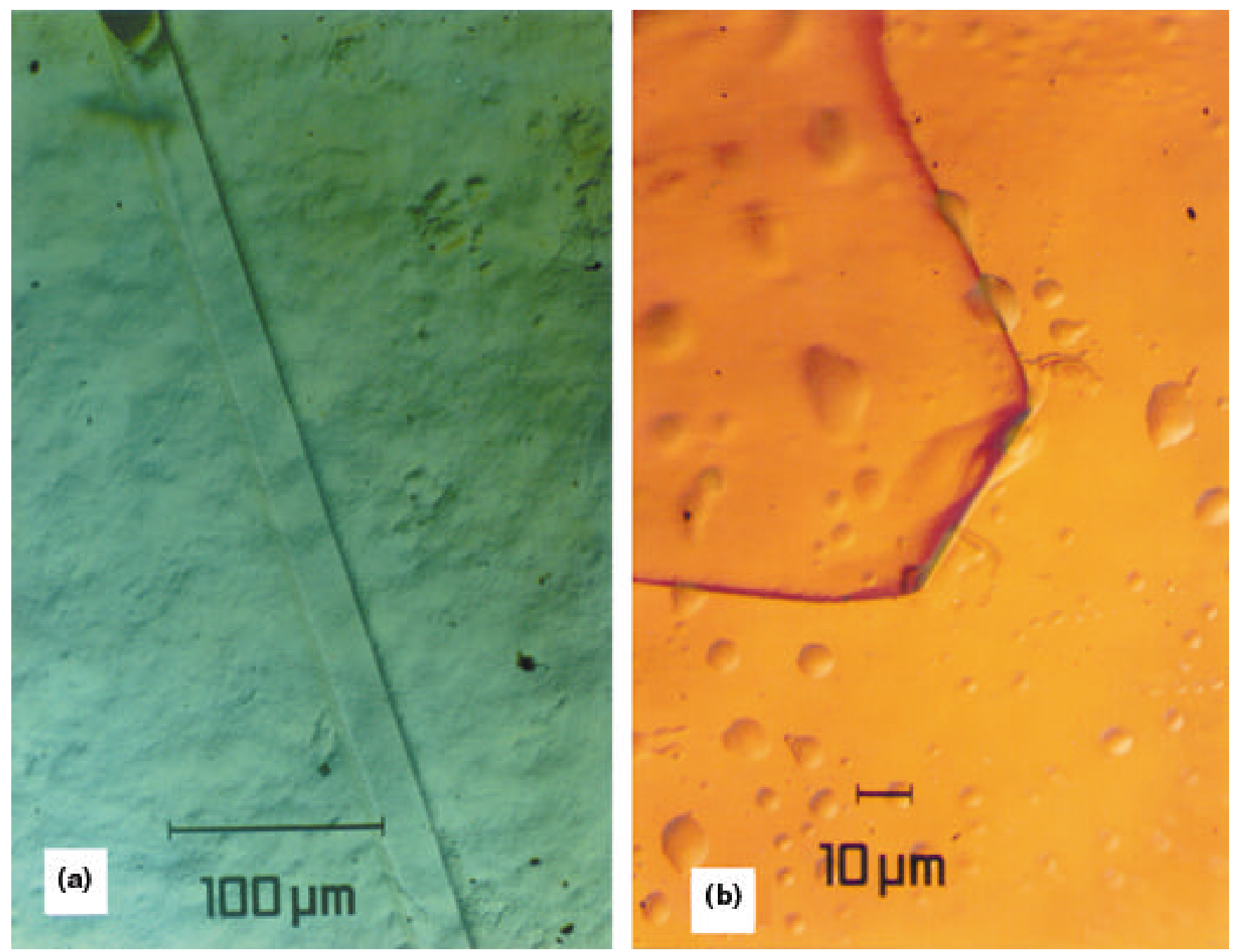

Figure 4. Optical microphotographs show a. twin boundary (lamellae) and b. grain boundary in a stoichiometric InSb crystal. This microstructure was developed as the intrinsic crystallographic micro-feature during crystal growth. 
sections of eutectic regions are seen with accompanying striation (figure 5b). Growth morphology feature's cause may be rotational vibration, convective instability, and temperature variations. However, these causes are responsible for the termination in layered growth and also affect the interface shape in succeeding growth. Other parameters of the microstructure are balance between radial and axial heat flow, growth rate and interface unstability. These structures may be generated by either rapid cooling or sudden increase in growth rate. In our growth configuration, heat loss (radiation) flux is radial as well as axial in growth chamber. It is also cited in literature (Rosenberger 1993; Overfelt et al 1995; Kuppalau 1997).

Figures $6 \mathrm{a}, \mathrm{b}$ show the minor phase growth in InSb16 and $\mathrm{GaSb} 3$ growth respectively with drastic reduction in aforementioned microstructures (twin, grain and dislocation etc). However, this is possible by controlling growth rate, temperature gradient and ampoule cone angle. Experimentally it is possible to reduce defects density significantly. Figure $6 a$ is seen with coalescence in
InSb16-4. The coalescence termination and irregularly shaped cross-section are indicative of the dynamic of solidification as the crystal-melt interface continuously trying to maintain stability in correlation to a micro fluctuating solidification rate of the TPB. Generally, higher density of smaller diameter coalescence shows increased solidification rate, while low-density of larger diameter coalescence represents decreased solidification rate corresponding to the termination or growth of coalescence due to the acceleration or deacceleration of growth rate (solidification) at the interface. Uniform coalescence in figure $6 \mathrm{a}$ is related to the lateral redistribution of the compositional field in the melt ahead of the homogeneous solidification front by diffusion. Branching in figure $6 \mathrm{a}$ showed that the growth rate is sufficiently low as to allow the lateral diffusion motion across the crystal-melt interface. Here, faceting phase leads to uncoupled growth of interface and resulted into irregular interface shape microscopically as seen in transverse micro-section (see figure $6 a)$.
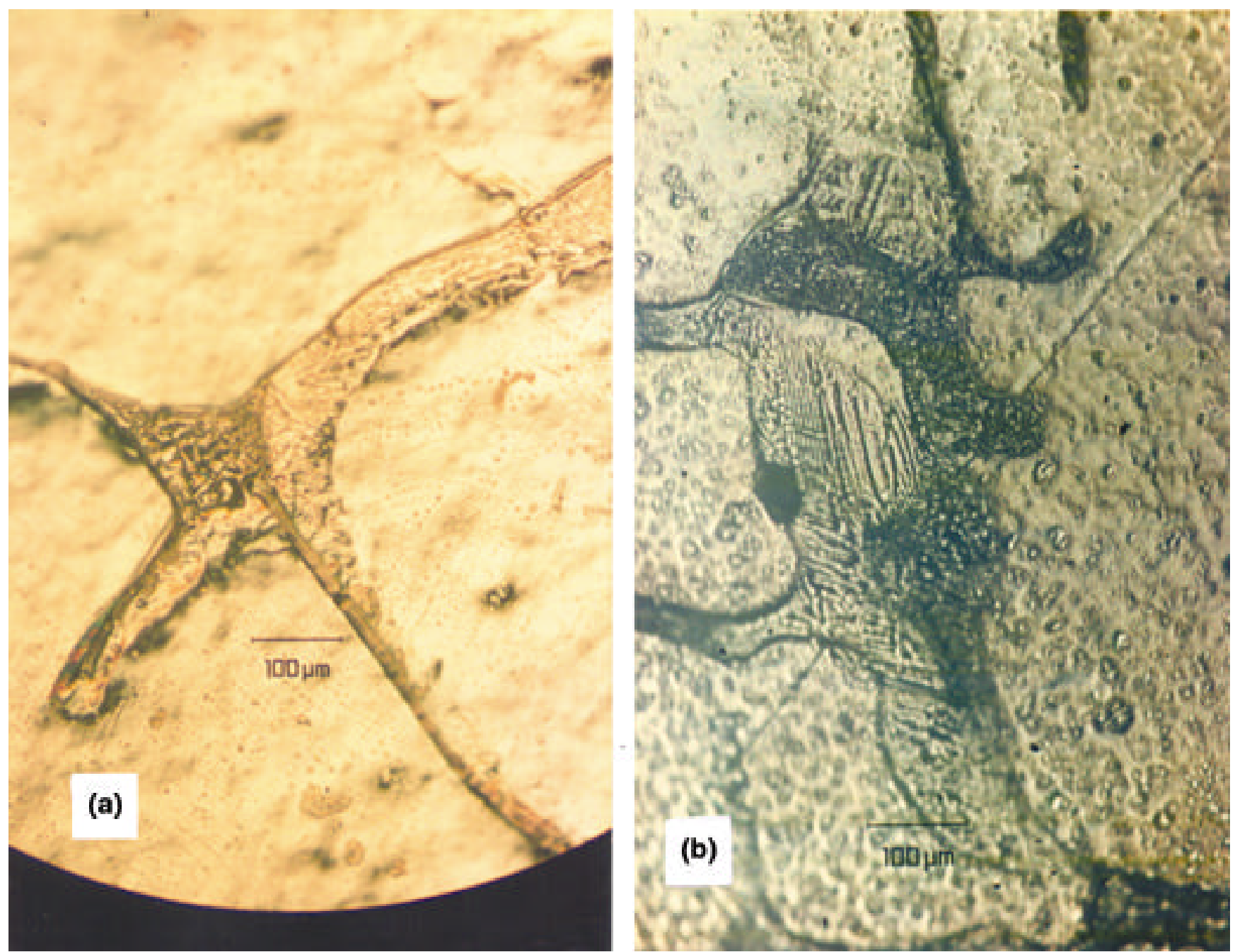

Figure 5. Microphotographs represent a. the eutectic star shaped microstructure in undoped InSb when excess Sb is incorporated and b. heavily Te-doped InSb growth showed striation near the off-eutectic growth. 

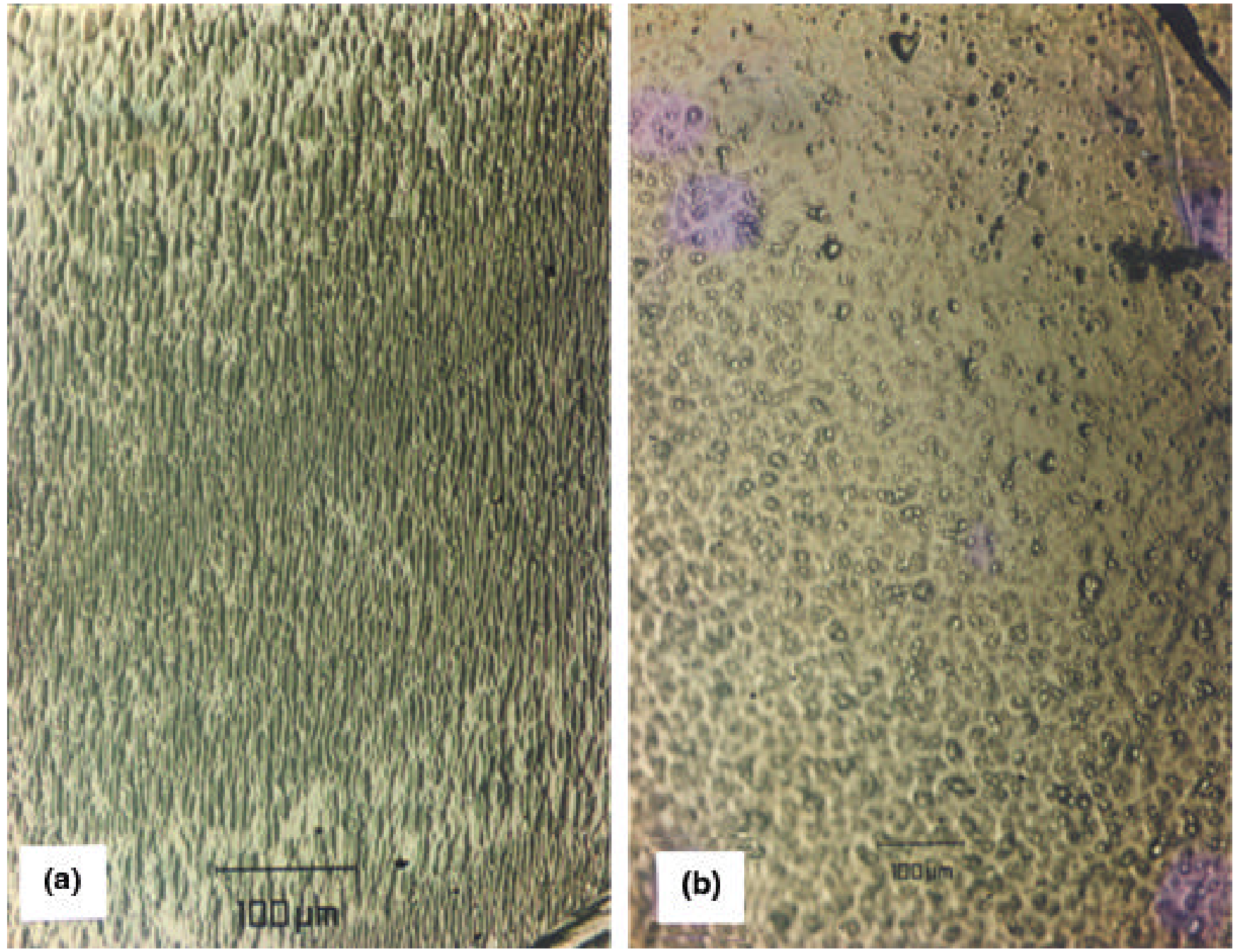

Figure 6. The minor phase effects are seen as a. the coalescence due to the micro irregular interface shape and b. a quasi-regular and uniform micro feature in single crystal growth in a optimized VDS technique.

Figure $6 \mathrm{~b}$ represents quasi-regular morphology or uniform micro features besides small irregularities are evident on the surface of the growing layer. However, it is not clear which phase leads in the growth. Without promoting facet phase, quasi-regular morphology is favoured at interface. Normally, branching, coalescence and quasiregular microstructures are related to the minor phase, which may be due to the diffusion of some species. This correspondence to ratio of temperature gradient $(\mathrm{d} z / \mathrm{d} T)$ to the solidification rate $(V g)$ should be minimum so that stable, planar growth would be propagated. In addition, crystal-melt interface shape must remain steady, continuous and uniform, which will result into single crystal growth. In VDS technique, we are very close to the optimum requirement of growth conditions required for the stability and shape at the interface.

\section{Conclusions}

In VDS technique, growth parameters and conditions are optimized for the single crystal growth. The quality of the crystal is influenced by the stability at interface and selfseeding. Influence of the interface shape on self-seeding i.e. single nucleation, has been studied and it is related to the crystalline quality of as grown ingots. VDS technique has promoted the self-seeding and preferred orientation growth with the significant reduction in micro defects. Therefore, this technique has been proposed for the growth of single crystal semiconductors.

\section{Acknowledgements}

The authors would like to thank Prof. S B Patel (UDP) for the help during growths. They also express their thanks to Prof. K S Chandrasekaran for the XRD measurements.

\section{References}

Althaus M, Sonnenberg K, Kussel E and Nacven R $1996 \mathrm{~J}$. Cryst. Growth 166622 
Aman J, Zemke D, Hoffmann B and Muller G 1996 J. Cryst. Growth 166646

Boiten P, Giacometti N, Santailler J L, Duerer T and Nobert J P 1998 J. Cryst. Growth 19443

de Oliveira C E M and de Carvalho M G 1995 J. Cryst. Growth 1519

Derebail R, Wilcox W R and Regel L L 1992 J. Cryst. Growth 11998

Gadkari D B, Lal K B, Shah A P and Arora B M 1997 J. Cryst. Growth 173585

Gadkari D B, Lal K B and Arora B M 1998a Bull. Mater. Sci. 21127

Gadkari D B, Lal K B, Gokhale N A, Shashidharan P, Shah A P and Arora B M 1998b Semiconductor materials (ed.) R K Bedi (Amritsar: Guru Nanak Dev Univ.) p. 144

Gadkari D B, Lal K B and Arora B M 1999a Indian Patent Appl. PBO/C-3 No. 3410/4159

Gadkari D B, Lal K B and Arora B M 1999b Fall meeting (Warrendale, PA: MRS) p. E10.29
Gadkari D B, Lal K B, Gokhale N A, Shashidharan P, Shah A P and Arora B M 1999c Indian J. Pure \& Appl. Phys. 37652

Hermann F M and Muller G 1995 J. Cryst. Growth 156 350

Ivanov Y M 1998 J. Cryst. Growth 194309

Kuppalau S K 1997 J. Cryst. Growth 172350

Lan C W and Ting C C 1995 J. Cryst. Growth 149175

Lopez C R, Mileham J R and Abbaschian R 1999 J. Cryst. Growth 2001

Overfelt R A, Matlock C A and Wilcox R C 1995 J. Cryst. Growth 147403

Marie Meyer (ed.) 1998 Compound semiconductors (Beverly, MA, USA: Franklin) 4 p. 4

Neugebauer P and Wilcox W R 1991 Acta Astronautica 25357

Rosenberger F 1993 Prog. Cryst. Growth \& Charact. 2687

Suzuli F, Okano Y, Hoshikawa F and Fukuda T 1993 J. Cryst. Growth 128435

Thomas R N, Hobyard H M, Ravishankar P S and Broggins T T 1993 Prog. Cryst. Growth \& Charact. 26219 